\title{
Fenología reproductiva de la queñua (Polylepis incarum, Rosaceae) durante un ciclo anual en la puna mesofítica de La Paz, Bolivia
}

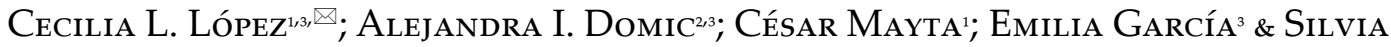 \\ C. GALlegos 33
}

${ }^{1}$ Carrera de Biología, Universidad Mayor de San Andrés. La Paz, Bolivia. ${ }^{2}$ Departamento de Antropología, The Pennsylvania State University, University Park, Pensilvania, EE.UU. ${ }^{3}$ Herbario Nacional de Bolivia, Instituto de Ecología, Universidad Mayor de San Andrés. La Paz, Bolivia. ${ }^{4}$ Martin-Luther Halle-Wittenberg University, Germany.

\begin{abstract}
Resumen. En el presente estudio se evaluó la fenología de la queñua (Polylepis incarum, Rosaceae) durante un ciclo anual en la puna mesofítica de La Paz, Bolivia. Esta especie es endémica de la cuenca del Lago Titicaca y, al igual que otras especies del género, $P$. incarum está categorizada como En Peligro para Bolivia debido a actividades antrópicas como la extracción de leña y la expansión de cultivos. Durante un año (marzo 2015 - febrero 2016) se realizaron evaluaciones mensuales en un parche de bosque en la comunidad de Kopacati, departamento de La Paz, Bolivia. Los objetivos del estudio fueron identificar las épocas de floración y fructificación, y relacionar el éxito reproductivo con el tamaño de la planta y con factores ambientales. $P$. incarum produce botones florales entre julio y agosto. La producción de flores en estado femenino se produce entre julio y noviembre, y la de flores en estado masculino, entre julio y octubre. La mayor producción de frutos inmaduros ocurrió a partir de noviembre; los frutos alcanzaron la madurez entre de diciembre y enero. Tanto la precipitación como la temperatura mostraron una influencia significativa en la producción de flores y frutos. El diámetro a la altura del pecho (DAP) fue el único carácter alométrico que influyó de manera significativa en la producción de flores y de frutos. No se detectaron diferencias significativas en la producción de flores y frutos entre árboles y arbustos. Los patrones fenológicos informados para esta especie son similares a los reportados para P. tomentella y $P$. besseri. Los resultados de este trabajo contribuyen al conocimiento de la biología reproductiva de Polylepis incarum y a la promoción de programas de conservación y reforestación de la especie.
\end{abstract}

[Palabras clave: biología reproductiva, Copacabana, floración, fructificación]

Aвstract. Reproductive phenology of queñua (Polylepis incarum, Rosaceae) during an annual cycle in the mesophytic puna of La Paz, Bolivia. In this study, the phenology of queñua (Polylepis incarum, Rosaceae) was evaluated during an annual cycle in the mesophytic puna of La Paz. The species is endemic to the Lake Titicaca basin. Like other species of the genus Polylepis, P. incarum is categorized as Endangered for Bolivia due to anthropic activities as firewood extraction and crop expansion. During one year (March 2015 - February 2016), we conducted monthly evaluations in a woodland located in the community of Kopacati, Copacabana department, La Paz, Bolivia. The objectives of the study were to identify flowering and fruiting times, and to assess the relationships between plant size, environmental factors and plant reproductive success. The species produces floral buds between July and August. The production of flowers in the female state occurs between July and November, and flowers in the male state, between July and October. The highest production of immature fruits took place in November, reaching maturity in December and January. Both precipitation and temperature showed a significant influence on flowers and fruits production. Diameter at breast height $(\mathrm{DBH})$ was the only allometric character significantly associated to the production of flowers and fruits between trees and shrubs. There was no significant difference in the production of flowers and fruits between trees and shrubs. The phenological patterns informed for this species are similar those reported for $P$. tomentella and $P$. besseri. The results of the present study contribute to the knowledge about the reproductive biology of Polylepis incarum and to promote programs of conservation and reforestation of the species.

[Keywords: reproductive biology, Copacabana, flowering, fruiting]

Editora asociada: Erica Cuyckens

$\triangle$ cecilia.lopez.alipaz@gmail.com 


\section{INTRODUCCIÓN}

La fenología es un aspecto fundamental en la ecología de las plantas. La producción de flores en determinadas épocas del año puede influir en la relación planta-animal en un ecosistema (Cleland et al. 2007; Petanidou et al. 2014) y también en la dinámica poblacional de una especie debido a su relación con la reproducción (Batalha and Martins 2004). La floración puede determinar el éxito reproductivo de las plantas; una floración tardía podría afectar la maduración de las semillas y una floración temprana podría verse desfavorecida por la baja cantidad de polen. Los estudios fenológicos permiten determinar la temporalidad de la foliación, la floración y la fructificación en un ciclo anual (Arteaga 2007; Cleland et al. 2007), con lo cual se puede identificar la época de floración y fructificación para planificar épocas de colecta de semillas, detección de árboles semilleros y las mejores fuentes de germoplasma (Domic 2012).

La floración y la fructificación están determinadas por factores ambientales y genéticos (Wagner et al. 2014). Entre los factores ambientales se encuentran las horas luz/oscuridad, la temperatura y la precipitación (Cruz-Hernández etal. 2002). Los factores ambientales pueden influir en la época del año en la que se produce la floración y su duración (Inouye et al. 2003). Se espera que los patrones ambientales se modifiquen debido al cambio climático, lo que ocasionará cambios en la precipitación y la temperatura, que, a su vez, podrían modificar la fenología de las plantas (Cleland et al. 2007). Sin embargo, se necesitan estudios que determinen el grado de relación entre la fenología de una especie y los distintos factores ambientales para analizar los posibles cambios en los patrones fenológicos frente a diferentes escenarios climatológicos (Cleland et al. 2007). Además de los factores ambientales, la floración y la fructificación se pueden relacionar con características morfológicas de las plantas (e.g., el tamaño, el diámetro a la altura del pecho [DAP] o el ancho del dosel [Wallace and Painter 2003]). Algunas especies pueden presentar individuos arbustivos y arbóreos. Este es el caso de las plantas del género Polylepis. En $P$. tomentella se observó que existen diferencias en la producción de flores y frutos entre las dos formas de crecimiento. Si bien los arboles producen más flores y frutos, la época en la que florecen y fructifican es la misma (Domic et al. 2013).
La queñua o kewiña, nombre común con el que se conoce al género Polylepis, se encuentra distribuida sólo en los Andes tropicales y subtropicales de Sudamérica, y forma los bosques naturales a mayor altitud en el mundo (Kessler and Schmidt-Lebuhn 2006; Vega et al. 2016). Estos bosques son uno de los ecosistemas más vulnerables de los altos Andes y del mundo, tanto por el ambiente donde se desarrollan como por la presión humana creciente (Kessler and SchmidtLebuhn 2006; Vega et al. 2016). En Bolivia existen 14 especies de Polylepis (Kessler and Schmidt-Lebuhn 2006), entre las cuales $P$. incarum tiene una distribución restringida para Bolivia y partes del departamento de Puno, en Perú (Montesinos et al. 2015). P. incarum forma mayormente fragmentos boscosos (Fernández et al. 2001) muy amenazados por actividades antrópicas como la extracción de leña, los incendios, el sobrepastoreo y la agricultura (Fjeldså and Kessler 2004; Mendoza and Cano 2011). Estas actividades generaron la reducción de extensas áreas boscosas y, posiblemente, cambios en los patrones de regeneración (Montesinos et al. 2015). En consecuencia, esta especie fue recategorizada como En Peligro (EN) para Bolivia en el Libro Rojo de la Flora Amenazada de Bolivia (Arrázola 2011). Existen grandes vacíos de información sobre la biología y la ecología de $P$. incarum, además de información básica sobre su biología reproductiva. El estudio de la fenología reproductiva de la especie podría apoyar a los programas de conservación y reforestación al identificar los meses ideales para colectar semillas y las características alométricas de las plantas semilleras (Henríquez et al. 2012). Los objetivos del presente estudio fueron: 1) evaluar el patrón fenológico de $P$. incarum en un ciclo anual en la comunidad de Kopacati, Copacabana, La Paz; 2) evaluar y comparar la producción y las épocas de floración y de fructificación entre árboles y arbustos de $P$. incarum, y 3) evaluar la influencia de factores ambientales y características alométricas en la producción de flores y frutos.

\section{Materiales y Métodos}

\section{Área de estudio}

El estudio se realizó en la comunidad de Kopacati, ubicada en el departamento de La Paz, Bolivia, $5 \mathrm{~km}$ al sur de Copacabana, en la provincia Manko Kapac, Cantón Locka, camino a Kasani (Figura 1). La temperatura media anual en el área de estudio es de $8{ }^{\circ} \mathrm{C}$, 
AREA DE ESTUDIO

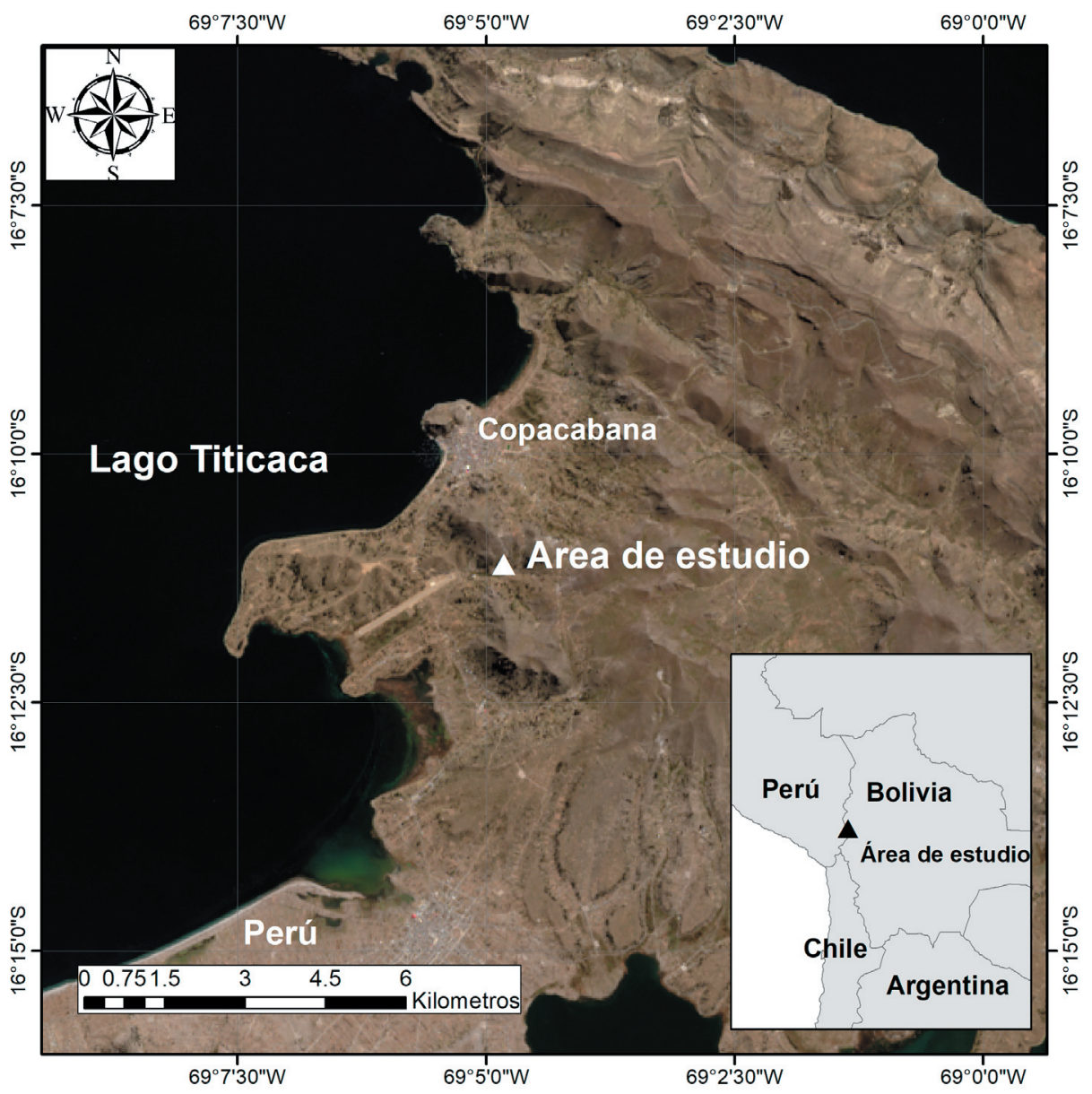

Figura 1. Area de estudio.

Figure 1. Study area.

con una temperatura diaria que oscila entre 15 y $20^{\circ} \mathrm{C}$, y que puede disminuir hasta $0^{\circ} \mathrm{C}$ en la noche (Paduano et al. 2003; SENAMHI). Esta región presenta un período seco entre abril y noviembre, y un período de lluvia entre diciembre y marzo. Este último representa 65$78 \%$ de la precipitación anual, que en promedio es $758 \mathrm{~mm} /$ año (Paduano et al. 2003). El bosque de $P$. incarum en el que se realizó el estudio tiene una extensión de 1.73 ha $\left(16^{\circ} 34^{\prime} 24^{\prime \prime} \mathrm{S}\right.$ y $68^{\circ} 04^{\prime} 52.5^{\prime \prime}$ O) y una elevación entre 3860 y $4082 \mathrm{~m} \mathrm{s.n.m}$. El suelo es predominantemente arcilloso, con $\sim 20 \%$ de pedregosidad. En el sector se pueden encontrar individuos de $P$. incarum como especie dominante, asociados a líquenes, helechos, crucíferas, vegetación arbustiva como Baccharis spp. y árboles del género Eucaliptus sp., además de vegetación herbácea, principalmente compuesta de gramíneas. En el área existe una intervención antrópica fuerte, con pastizales próximos a cultivos rodeados por cortinas rompevientos de troncos de árboles de queñua talados (Vega et al. 2016).

\section{Especie de estudio}

Polylepis incarum (Bitter) M. Kessler and Schmidt-Leb incluye árboles y arbustos de entre 1 y $10 \mathrm{~m}$ de altura (Fernández et al. 2001; Kessler and Schmidt-Lebuhn 2006). Las hojas poseen entre 1 y 2 pares de folíolos y se encuentran en mayor cantidad en las puntas de las ramas. Las flores son hermafroditas, con 0.5-1 cm de diámetro, entre 14 y 20 estambres de anteras orbiculares, y presentan dicogamia (Berry and Calvo 1989; Kessler 1995; Vega et al. 2007). Los frutos son aquenios alados, con espinas, que se dispersan por el viento.

\section{Diseño experimental}

En el bosque de Puma Kato se marcaron de forma permanente 80 individuos: 40 árboles y 40 arbustos, elegidos al azar. Cada 
individuo fue georreferenciado, marcado con placas de aluminio y cinta 'flagging' a la altura de las primeras ramas. Cada individuo fue evaluado una vez al mes durante un año (de marzo 2015 a febrero 2016). Todas las plantas fueron medidas y se registraron los siguientes caracteres alométricos: altura total, diámetro a la altura del pecho (DAP), altura del tronco y área del dosel. Para medir el DAP se usó un dasómetro y se obtuvo el diámetro de cada individuo. En el caso de los arbustos, se midió el diámetro de los troncos principales. Para todos los individuos, en cada evaluación se eligió una rama diferente ubicada en cada orientación cardinal. En cada una de estas ramas, considerando 30 cm desde la punta, se registró el número de estructuras reproductivas en las siguientes etapas fenológicas: a) botones florales, b) flores maduras en estadío femenino, c) flores en estadío masculino, d) frutos maduros, y e) frutos secos retenidos en la planta (Figura 2).

\section{Análisis de datos}

Para determinar las diferencias entre cada fenofase se realizó un análisis de producción máxima de cada fase reproductiva. A fin de establecer la relación de la producción de flores y frutos con las condiciones ambientales y los parámetros alométricos se realizó una serie de modelos lineales generalizados (GLM). Estos modelos fueron analizados por separado para árboles y arbustos (Domic et al. 2013). La sobredispersión de los modelos fue ajustada usando estimaciones de cuasi-verosimilitud (quasi-likelihood) con una función de enlace logarítmica. Para conocer la respuesta de la producción de flores y frutos a las condiciones ambientales y los parámetros alométricos, el modelo inicial incluyó todos los caracteres que se cuantificaron (altura total, ancho del dosel, DAP, temperatura promedio y precipitación), relacionados con el promedio de flores y frutos producidos, por separado. Los datos de precipitación y temperatura promedio de un ciclo anual fueron proporcionados por el Servicio Nacional de Meteorología e Hidrología (SENAMHI). Estos datos se obtuvieron de la estación meteorológica de Copacabana. Todos los análisis se realizaron en el ambiente $\mathrm{R}$ versión 3.2.5, con el paquete stats.

\section{Resultados}

En total, en los 80 individuos estudiados (40 árboles y 40 arbustos) se contabilizaron 39550 botones florales, 39561 flores femeninas, 39558 flores masculinas, 39622 frutos inmaduros y 39622 frutos maduros. La Figura 3 muestra el patrón fenológico observado en un ciclo anual de $P$. incarum. La producción de botones se observó entre junio y octubre, con un pico en el mes de agosto. La floración se produce entre julio y noviembre, y se alcanza la mayor producción de flores en septiembre. La mayor producción de frutos inmaduros fue observada entre agosto y enero, con un pico de producción en noviembre. La producción máxima de frutos maduros se detectó en diciembre, y se encontraron, ocasionalmente, hasta fines de marzo.

Al considerar la influencia de las variables ambientales en las fenofases, encontramos que la producción de botones y flores no muestra un patrón asociado con la temperatura media (Figura 4a, c). Sin embargo, observamos que la mayor parte de los individuos produjeron
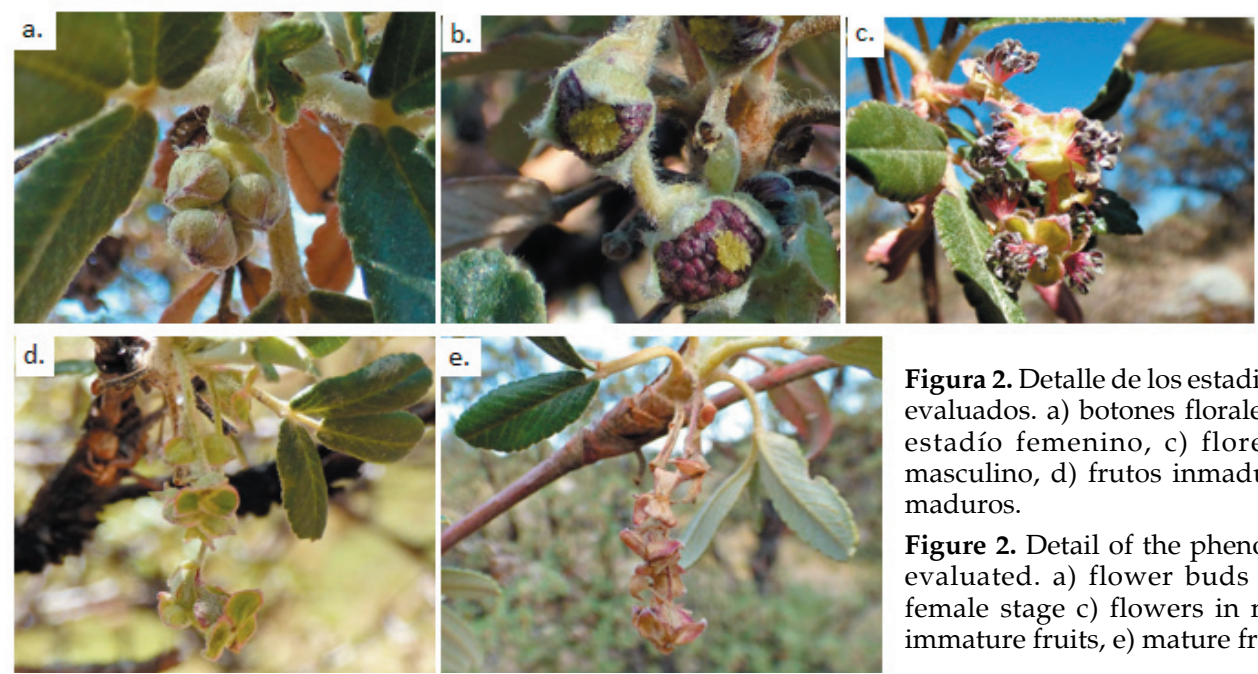

Figura 2. Detalle de los estadios fenológicos evaluados. a) botones florales, b) flores en estadío femenino, c) flores en estadío masculino, d) frutos inmaduros, e) frutos maduros.

Figure 2. Detail of the phenological stages evaluated. a) flower buds b) flowers in female stage c) flowers in male stage, d) immature fruits, e) mature fruits. 


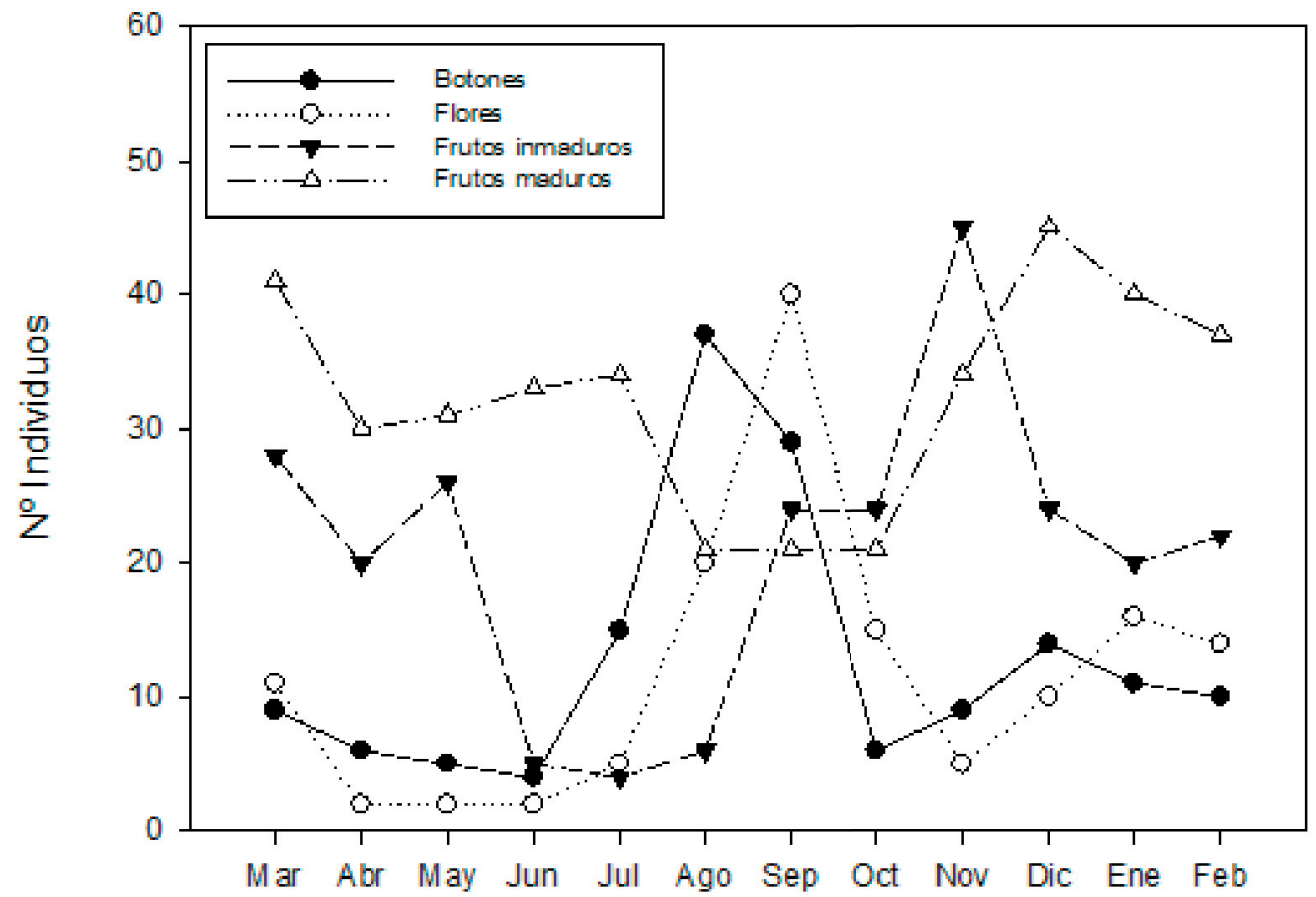

Figura 3. Detalle de las fenofases observadas de P. incarum en un ciclo anual: botones florales, flores, frutos inmaduros y frutos maduros.

Figure.3. Detail of the observed phenophases of P. incarum in an annual cycle: floral buds, flowers, immature fruits and mature fruits.

frutos durante los meses más calientes (Figura 4e). Considerando la precipitación, la mayor cantidad de individuos produjeron botones y flores durante la época seca, entre junio y septiembre (Figura $4 \mathrm{~b}$ y d), y la mayor cantidad de individuos produjeron frutos a inicios de la época húmeda, entre octubre y noviembre (Figura 4f).

La producción de botones se asoció significativamente con la precipitación total mensual, pero no con la temperatura promedio mensual (Tabla 1a). La producción de flores y frutos esta significativamente asociada con la temperatura media y con la precipitación total mensual (Tabla 1b y c). En todos los casos se observó que la precipitación afecta de manera negativa la producción de botones, flores y frutos, y una mayor producción cuando la precipitación es menor.

No se encontró una relación significativa entre el hábito y la producción de botones, flores o frutos (Tabla 2a). Al analizar el efecto de las variables alométricas en la producción de botones, flores y frutos se observó que el DAP es el único carácter asociado significativamente con la producción de botones, flores y frutos (Tabla 2). La altura $\mathrm{y}$ el dosel no tuvieron efectos significativos en la producción de botones, flores o frutos (Tabla 2b, c, d).

Tabla 1. Resultados del modelo lineal generalizado (GLM) de las variables ambientales consideradas sobre la producción de botones florales, flores y frutos.

Table 1. Results of the generalized linear model (GLM) of the environmental variables considered on flower buds, flowers and fruit production.

\begin{tabular}{lcccc}
\hline & Estimación & ES & Valor de tó z & $P$ \\
\hline $\begin{array}{l}\text { Producción de botones florales } \\
\quad \text { Temperatura }\end{array}$ & -0.018 & 0.098 & -0.186 & 0.852 \\
$\quad$ Precipitación & -0.007 & 0.002 & -3.613 & $<0.001$ \\
$\begin{array}{l}\text { Producción de flores } \\
\quad \text { Temperatura }\end{array}$ & 0.321 & 0.129 & 2.485 & $<0.05$ \\
$\quad$ Precipitación & -0.007 & 0.002 & -3.037 & $<0.001$ \\
Producción de frutos & & & & $<0.001$ \\
$\quad$ Temperatura & 1.056 & 0.090 & 11.611 & $<0.001$ \\
$\quad$ Precipitación & -0.010 & 0.001 & -7.063 & \\
\hline
\end{tabular}



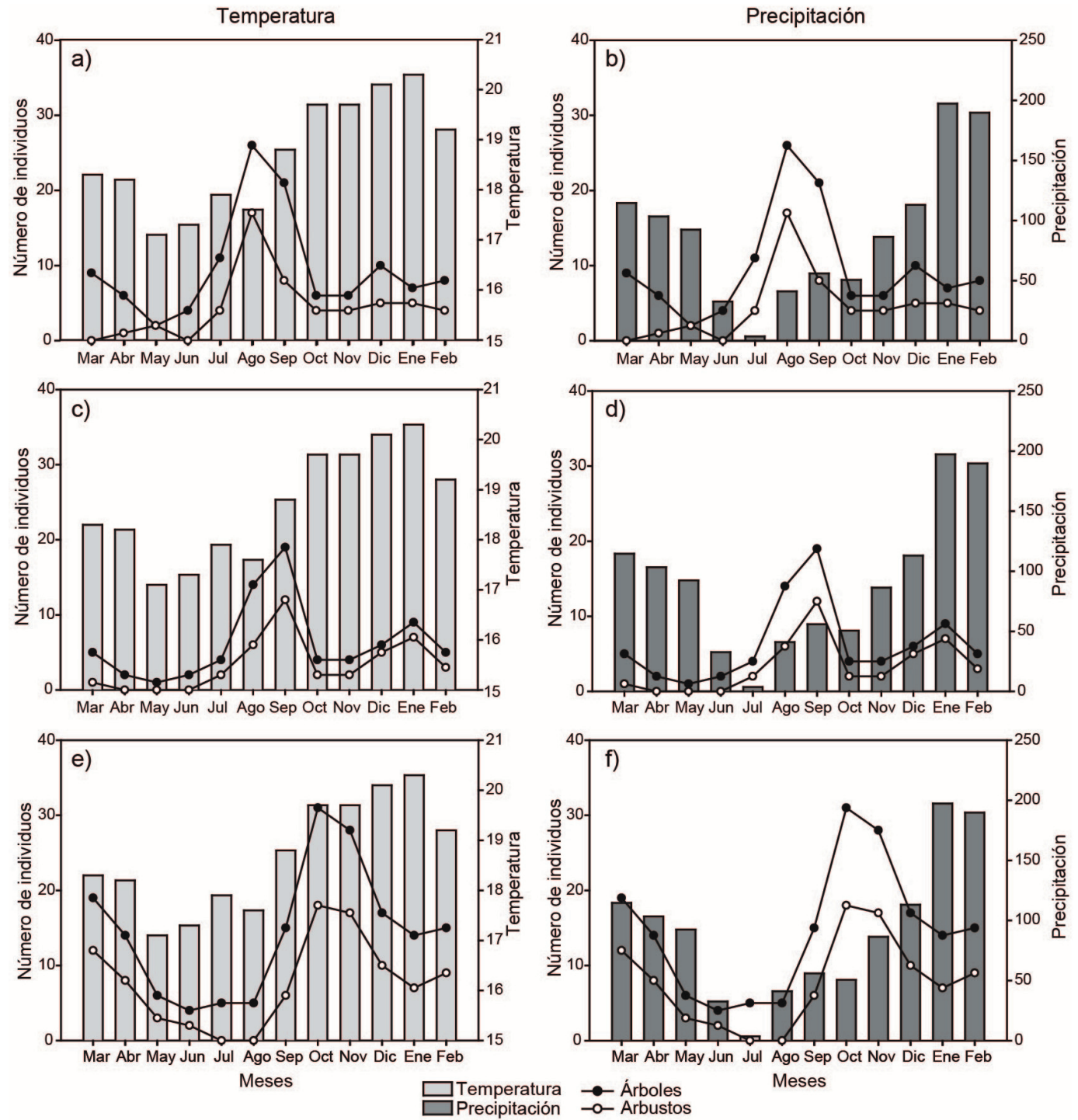

Figura 4. A la izquierda, relación de la producción de a) botones, c) flores, e) frutos, con la temperatura en P. incarum. A la derecha, relación de la producción de b) botones, d) flores, f) frutos con la precipitación.

Figure 4. On the left, the relation of the production of a) buttons, c) flowers, e) fruits, with temperature in P. incarum. On the right, the relation of the production of $b$ ) buttons, d) flowers, f) fruits with precipitation.

Tabla 2. Resultados del modelo lineal generalizado (GLM) del hábito y de las variables alométricas consideradas sobre la producción de botones florales, flores y frutos.

Table 2. Results of the generalized linear model (GLM) of the habit and the allometric variables considered on flower buds, flowers and fruit production.

\begin{tabular}{lcccc}
\hline & Estimación & FS & Valor de tó z & $P$ \\
\hline $\begin{array}{l}\text { Producción total por hábito } \\
\quad \text { Hábito }\end{array}$ & -6.263 & 3.877 & -1.615 & 0.111 \\
$\begin{array}{l}\text { Producción de botones florales } \\
\quad \text { DAP }\end{array}$ & 0.041 & 0.006 & 6.090 & $<0.001$ \\
$\quad$ Altura & 0.001 & 0.0009 & 1.331 & 0.183 \\
$\quad$ Ancho del dosel & -0.0008 & 0.0007 & -1.172 & 0.241 \\
Producción de flores & 0.026 & 0.010 & 2.533 & $<0.05$ \\
$\quad$ DAP & 0.0009 & 0.001 & 0.69 & 0.487 \\
$\quad$ Altura & -0.0002 & 0.001 & -0.275 & 0.783 \\
$\quad$ Ancho del dosel & & & & $<0.001$ \\
Producción de frutos & 0.036 & 0.005 & 6.831 & 0.111 \\
$\quad$ DAP & 0.001 & 0.0007 & 1.595 & 0.139 \\
$\quad$ Altura & 0.0008 & 0.0005 & -1.482 & \\
$\quad$ Ancho del dosel & & &
\end{tabular}




\section{Discusión}

El patrón fenológico observado en Polylepis incarum se caracteriza por la floración durante la época seca y comienzos de la época húmeda, específicamente entre julio y noviembre. Este patrón es similar al reportado para otras especies del género como $P$. tomentella, que florece entre junio y octubre (Domic et al. 2013) y P. besseri, que florece entre junio y agosto (Hensen 1994). Sin embargo, existen especies de este género que florecen en meses diferentes, como P. australis, que florece entre septiembre y noviembre (Seltmann et al. 2009).

La fenología de las plantas está influenciada por variables ambientales como las horas luz, la temperatura, la precipitación y la radiación, entre otros (Bustamante and Búrquez 2008). En este estudio se observó la presencia de botones florales y flores entre junio y noviembre, que es la época de menor precipitación. Según varios autores, se requiere estrés hídrico para estimular el desarrollo de flores (OrduzRodríguez and Fischer 2007). En ambientes estacionales tropicales, las plantas leñosas pueden iniciar su floración en la estación seca. Por ejemplo, Jacquinia aristata y Pereskia guamacho, dos especies arbóreas distribuidas en regiones semiáridas de Venezuela, comienzan la floración en estaciones secas (LemusJiménez and Ramírez 2002). Otras plantas leñosas presentes en regiones semiáridas de México (e.g., Prosopis laevigata) también inician la floración en época seca (Pavón and Briones 2001). Otro factor ambiental que podría influir en la producción de flores es el viento, ya que las especies de Polylepis dependen de éste para su polinización y dispersión de semillas (Seltmann et al. 2007). En lugares como las regiones montañosas de Córdoba, donde se encuentra P. australis (Seltmann et al. 2007), los meses de septiembre y noviembre se caracterizan por el incremento de la velocidad del viento (Zilio and Zilio 2007). La floración de $P$. incarum, $P$. tomentella y $P$. besseri también coinciden con las épocas de mayores vientos (Kessler and Schmidt-Lebuhn 2006).

Los Andes centrales presentan una estacionalidad climática muy marcada, con dos épocas bien distinguibles: la seca y la húmeda. En la puna mesofítica sureña, donde habita P. incarum, la temperatura aumenta en la época seca (Navarro 2002). La fructificación de $P$. incarum, que se produce entre septiembre y diciembre, es similar a las de $P$. besseri y $P$. tomentella, que ocurren al inicio de la época de lluvias (Hensen 1994; Domic et al. 2013). En nuestro estudio, la producción de frutos de $P$. incarum estuvo influenciada por la precipitación y la temperatura. Este proceso se inicia al final de la época seca y coincide con el aumento de temperatura. La fructificación al principio de la época de lluvias podría ser una estrategia para estimular la germinación y el desarrollo de plántulas en suelo húmedo (Domic et al. 2013). La temperatura también influyó en la fructificación de P. incarum; Saravia and Vintimilla (2016) describen que para $P$. reticulata, la temperatura es un factor que influye en el desarrollo de flores y frutos. La formación de estas estructuras requiere de varios días de temperaturas favorables. Esta podría ser la razón por la cual $P$. incarum fructifica cuando la temperatura aumenta.

En cuanto a los caracteres alométricos, el DAP fue el único que influyó significativamente en la producción de flores y frutos de $P$. incarum. Una mayor producción de flores y frutos en plantascon mayor diámetrotambién seobservó en otras especies, donde el DAP influía de forma positiva en el número de inflorescencias (Queenborugh et al. 2013) y en la producción de frutos (Adler and Kielpinski 2000). No se encontró una diferencia significativa en la producción de flores y frutos entre árboles y arbustos. Este resultado difiere al observado en $P$. tomentella; en esta especie, los árboles producen mayor cantidad de flores y frutos (Domic et al. 2013). Los árboles y arbustos de las dos especies florecen y fructifican en la misma época.

El número creciente de estudios en bosques de Polylepis refleja la importancia de conservar estos ecosistemas (Martínez and Villarte 2009). El conocimiento sobre la biología reproductiva de $P$. incarum permitirá implementar estrategias de conservación y regeneración de estos parches de bosque que se encuentran en peligro de extinción junto a toda su flora y fauna asociada. Los datos que se reportan en el presente trabajo permitirán definir las mejores épocas de colecta de frutos, además de identificar a los individuos que producirán más semillas. Es importante continuar con estudios que favorezcan la preservación de estos bosques. Por esta razón recomendamos continuar con estudios que identifiquen otros factores determinantes de la fenología de $P$. incarum.

Cabe señalar que los patrones de floración y fructificación obtenidos en este trabajo deben ser corroborados, ya que las observaciones se 
realizaron durante un ciclo anual. Si bien el estudio cubre un corto período de tiempo, consideramos importantes los trabajos con esta especie debido al estado de conservación y a la carencia de información sobre la biología reproductiva de $P$. incarum. Actualmente, la especie está categorizada como En Peligro para Bolivia, y las poblaciones remanentes se encuentran amenazadas por la expansión veloz de cultivos agrícolas y asentamientos urbanos. Recientemente se ha planteado que se debería reclasificar a $P$. incarum como En Peligro Crítico por su estructura poblacional y su reducida área de ocupación (Domic et al. 2017). Hoy en día, no existen programas de conservación ni de reforestación enfocados en P. incarum. Este estudio podría apoyar con información básica para identificar etapas de colectas de semillas para la producción de plantines para recuperar sus poblaciones.

Agradecimientos. Queremos agradecer al departamento de Biotecnología Vegetal del Instituto de Biología Molecular y Biotecnología, al proyecto "Germinación de especies del género Polylepis para la producción de plantines, destinada a la reforestación de las áreas verdes urbanas del Municipio de La Paz y su revaloración como especie nativa", a Jorge Quezada, Juan Carlos Bermejo, Cecilia Vega y Paola Rocabado. De la misma manera al Instituto de Ecología de la Universidad Mayor de San Andrés por todo el apoyo financiero para la realización del trabajo.

\section{REFERENCIAS}

Adler, G. H., and K. A. Kielpinski. 2000. Reproductive phenology of a tropical canopy tree Spondias mombin. Biotropica 32:686-69.

Arrázola, S. 2011. Polylepis incarum (Bitter) M. Kessler and Schmidt-Leb. Pp. 44-45 en Libro rojo de la flora amenazada de Bolivia Volumen I - Zona Andina. MMAyA. La Paz.

Arteaga, L. 2007. Fenología y producción de semillas de especies arbóreas maderables en un bosque húmedo montano de Bolivia. (PN ANMI Cotapata). Revista Boliviana de Ecología 21:57-68.

Batalha, M., and F. Martins. 2004. Reproductive phenology of the cerrado plant community in Emas National Park (central Brazil). Australian Journal of Botany 52:149-161.

Berry, P., and R. Calvo. 1989. Wind pollination, self-incompatibility, and altitudinal shifts in pollination systems in the high Andean genus Espeletia (Asteraceae). American Journal of Botany 76:1602-1614.

Bustamante, E., and A. Búrquez. 2008. Effects of Plant Size and Weather on the Flowering Phenology of the Organ Pipe Cactus (Stenocereus thurberi). Annals of Botany 102:10019-1030.

Cleland, E., I. Chuine, A. Menzel, H. Mooney, and M. Schwartz. 2007. Shifting plant phenology in response to global change. Trends in Ecology and Evolution 22(7):357-365.

Cruz-Hernández, O., A. Martínez-Rodríguez., S. Cruz-Izquierdo, and L. Serrano-Covarrubias. Determinación de la duración de diferentes procesos fenológicos del durazno "oro de Tlaxcala" y su potencialidad de producción en Altzayanca, Alaxcala. Revista Chapingo Serie Zonas Áridas 3(1):35-40.

Domic, A. 2012. Effects of anthropogenic disturbances on the regeneration of an Andean tree species. PhD thesis, Saint Louis University. USA. Pp. 170.

Domic, A., E. Mamani, and G. Camilo. 2013. Fenología reproductiva de la kewiña (Polylepis tomentella, Rosaceae) en la puna semihúmeda de Chuquisaca (Bolivia). Ecología en Bolivia 48(1):31-45.

Domic, A. I., A. N. Palabral-Aguilera, M. I. Gómez, R. Hurtado, N. Ortuño, and M. Liberman. 2017. Polylepis incarum (Rosaceae) una especie En Peligro Crítico en Bolivia: Propuesta de reclasificación en base al área de ocupación y estructura poblacional. Ecología en Bolivia 52(2):53-68.

Fernández, M., M. Mercado, S. Arrázola, and E. Martínez. 2001. Estructura y composición florística de un fragmento boscoso de Polylepis besseri Hieron subsp. besseri en Sacha Loma, Cochabamba. Revista Boliviana de Ecología 9:1527.

Fjeldså, J. 2002. Polylepis forest- vestiges of a vanishing ecosystem in the Andes. Ecotropica 8:111-123.

Fjeldså, J., and M. Kessler. 2004. Conservación de la biodiversidad de los bosques de Polylepis de las tierras altas de Bolivia. Una contribución al manejo sustentable en los Andes. DIVA Technical Report 11, Editorial FAN, Santa Cruz. Pp. 214.

Henríquez, C., G. Sotes, and R. Bustamante. 2012. Fenología reproductiva de Pouteria splendens (Sapotaceae). Guyana Botánica 69(2):251-255.

Hensen, I. 1994. Estudios ecológicos y fenológicos sobre Polylepis besseri Hieron en la Cordillera Oriental boliviana. Ecología en Bolivia 23:21-32.

Inouye, D., F. Saavedra, and W. Lee-Yang. 2003. Environmental influences on the phenology and abundance of flowering by Androsace septentrionalis (Primulaceae). American Journal of Botany 90:905-910.

Kessler, M. 1995. The genus Polylepis in Bolivia. Candollea 50:131-170.

Kessler, M., and A. N. Schmidt-Lebuhn. 2006. Taxonomical and distributional notes on Polylepis (Rosaceae). Organisms Diversity and Evolution 6:67-69.

Lemus-Jiménez, L., and N. Ramírez. 2002. Fenología reproductiva en tres tipos de vegetación de la planicie costera de 
la península de Paraguana, Venezuela. Acta Científica Venezolana 53:266-278.

Martínez, O., and F. Villarte. 2009. Estructura dasométrica de las plantas de un parche de Polylepis besseri incarum y avifauna asociada en la Isla del Sol (Lago Titicaca, La Paz - Bolivia). Ecología en Bolivia 44(1):36-49

Mendoza, W., and A. Cano. 2011. Diversidad del género Polylepis (Rosaceae, Sanguisorbeae) en los Andes peruanos. Revista Peruana de Biología 18:197-200.

Montesinos, D., A. Pinto, D. Beltrán, and W. Galiano. 2015. Vegetación de un bosque de Polylepis incarum (Rosaceae) en el departamento de Lampa Puno, Perú. Revista Peruana de Biología 22:87-96.

Navarro, G. 2002. Vegetación y unidades biogeográficas. En: G. Navarro y M. Maldonado, Geografía ecológica de Bolivia: Vegetación y ambientes acuáticos. Editorial Centro de Ecología Simón I. Patiño-Departamento de Difusión. Cochabamba, Bolivia. Pp. 719.

Orduz-Rodríguez, J., and G. Fischer. 2007. Balance hídrico e influencia del estrés hídrico en la inducción y desarrollo floral de la mandarina 'Arrayana' en el piedemonte llanero de Colombia. Agronomía Colombiana 25(2):255-263.

Paduano, G., M. B. Bush, P. A. Baker, S. C. Fritz, and G. O. Seltzer. 2003. A vegetation and fire history of Lake Titicaca since the Last Glacial Maximum. Palography, Paleoclimatology, Paleoecology 194:259-279.

Pavón, N., and O. Briones. 2001. Phenological patterns of nine perennial plants in an intertropical semi-arid Mexican scrub. Journal of Arid Environments 49:265-277.

Petanidou, T., A. Kallimanis., S. Sgardelis, A. Mazaris, J. Pantis, and N. Waser. 2014. Variable flowering phenology and pollinator use in a community suggest future phenological mismatch. Acta Oecologica 59:104-111.

Queenborugh, S., A. Humphreys, and R. Valencia. 2013. Sex-specific flowering patterns and demography of the understorey rain forest tree Irianthera hostmannii (Myristicaceae). Tropical Conservation Science 5:637-652.

Saravia, J., and D. Vintimilla. 2016. Actividad vegetativa, tasas de crecimiento y estomación de la edad de los individuos en los rodales de Polylepis reticulata del Parque Nacional Cajas. Tesis de grado previo a la obtención del título de Ingeniero Ambiental. Universidad de Cuenca, Cuenca. Pp. 115.

Seltmann, P., A. Cocucci, D. Renison, A. Cierjacks, and I. Hensen. 2007. Mating system, outcrossing distance effects and pollen availability in the wind-pollinated treeline species Polylepis australis BITT. (Rosaceae). Basic and Applied Ecology 10:52-60.

Seltmann, P., I. Hensen, D. Renison, K. Wesche, S. Ploch, J. R. Dueñas, A. Cocucci, and K. Jung. 2009. Biparental inbreeding depression, genetic relatedness and progeny vigour in a wind-pollinated treeline species in Argentina. Plant Ecology 205:155-164.

Servicio Nacional de Meteorología (SENAMHI). 2017. Consulta boletines mensuales. Estación Copacati.

Vega, C., J. C. Bermejo, G. Villegas, J. Quezada, M. Aguilar, and E. Conde. 2007. Propagación masiva de Polylepis tomentella Weddell ssp. nana mediante técnicas de cultivo in vitro. Ecología en Bolivia 42:102-120.

Vega, C., J. C. Bermejo, P. Rocabado, G. Villegas, J. Quezada, and C. López. 2016. Proyecto IDH (2013-2014) “La keñua (Polylepis) árbol altoandino como alternativa ornamental para espacios verdes urbanos del Municipio de La Paz". Unidad de Biotecnología Vegetal del Instituto de Biología Molecular y Biotecnología. Carrera de Biología. Facultad de Ciencias Puras y Naturales de la Universidad Mayor de San Andrés. IMP. ROTEMBOL. 1 ed. La Paz, Bolivia. Pp. 147.

Wagner, M., D. Lundberg, D. Coleman-Der, S. Tringe., J. Dangl, and T. Mitchell-Olds. 2014. Natural soil microbes alter flowering phenology and the intensity of selection on flowering time in a wild Arabidopsis relative. Ecology Letters 17:717-726.

Wallace, R., and L. Painter. 2003. Metodologías para medir la Fenología de Fructificación y su Análisis con relación a los Animales Frugívoros. Ecología en Bolivia, serie metodológica 2:1-14.

Zilio, C., and M. Zilio. Revalorización del patrimonio geológico como recurso turístico de Capilla del Monte. 2007. IX Jornadas de Investigación del Centro de Investigaciones Geográficas y del Departamento de Geografía, La Plata. 